\section{New Theory}

ISSN: 2149-1402
35 (2021) 20-31

Journal of New Theory

https://dergipark.org.tr/en/pub/jnt

Open Access

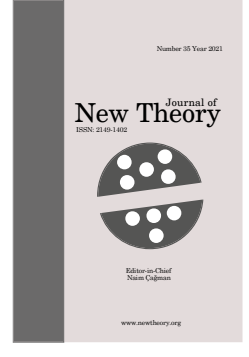

\title{
Fuzziness on Hopf Algebraic Structures with Its Application
}

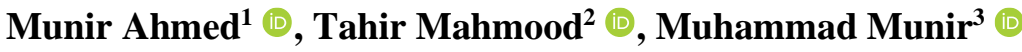

\begin{abstract}
Article History
Received: 01 May 2019

Abstract - The objective of writing this manuscript is to apply the concept of fuzzy set on some basic Hopf algebraic structures. In this manuscript, the novel concepts of fuzzy Hopf subalgebra, fuzzy Hopf ideal, and fuzzy $H$-submodule are proposed. Some properties of these concepts are discussed, and some

Accepted: 25 Jun 2021 significant results are also proved in it. The advantages of the proposed work are also studied in it. The

Published: 30 Jun 2021

Research Article application of the proposed work is also discussed in it.
\end{abstract}

Keywords - Fuzzy Hopf subalgebra, fuzzy Hopf ideal, fuzzy H-submodule

Mathematics Subject Classification (2020) - 03E72, 18M80

\section{Introduction}

Heinz Hopf did much work in the field of the algebraic topology of Lie groups [1,2]. Later on, mathematicians worked on it and named it after Heinz Hopf as Hopf algebra (HA) [3-5]. Vast applications of HA are in physics, quantum groups, non-commutative geometry and representation theory etc. [6,7], e.g., a particle move in space-time has HA structures [8]. So, it is necessary to extend these concepts in uncertainty. In 1969, Sweedler [9] wrote the first book on HA. After that, much research was done in this algebra [10,11].

HA is an algebra with dual structure coalgebra and has an endomorphism called antipode. In other words, we can also say that an algebra with the structures of cohomology and homology of a topological group is called HA. In 1939, such algebras were introduced and linked with Lie groups. An example of HA is the Steenrod algebra introduced in the 1960s by Milnor and Moore [12], the cohomology algebra.

Zadeh gave the approach to fuzzy sets (FS) in 1965 [13]. Rosenfeld worked on the notion of fuzzy groups [14]. Basically, in fuzzy sets, the range of membership degree is $[0,1]$, which tells us up to which degree the element belongs to a set. The fuzzy set theory deals with uncertainties. Fuzzy set theory is the door to developing an intelligent system for identification and decision making etc. There are vast applications of fuzzy sets in decision-making, pattern recognition, control theory, and optimization. A fuzzy set is also known as a model that represents uncertainty in the universe.

The thought of fuzzy submodule was given by Zahedi and Ameri [15]. Many authors used the concept of fuzzy submodules in different fields of mathematics and physics [16-18]. The thought of fuzzy subcomodule was offered by Chen and Akram [19] in 2012. Notions of comodule and coalgebra are generalizations and dualizations of module and algebra, respectively.

\footnotetext{
1irmunir@gmail.com; ${ }^{2}$ tahirbakhat@iiu.edu.pk; ${ }^{3}$ munir.phdma78@iiu.edu.pk (Corresponding Author)

${ }^{1}$ Islamabad Model College for Boys, F-10/4, Islamabad, Pakistan.

${ }^{2,3}$ Department of Mathematics \& Statistics, International Islamic University, Islamabad Pakistan
} 
The thought of fuzzy algebra was offered by Abdulkhalikov et al. [20] in 1988. The thought of fuzzy subcoalgebra was given by Chen [21] in 2009, and the thought of fuzzy subbialgebra was also given by Chen and Wenxu [22] in 2012. The concept of fuzziness was also applied in some other algebras in [23-25].

The main problem in mathematics containing uncertainty is how to carry out the ordinary concepts to the uncertainty case. The proposed work will help in dealing with uncertainty problems in HA and quantum groups. Our obtained results probably can be applied in various fields such as artificial intelligence, signal processing, multiagent systems, pattern recognition, robotics, expert systems, medical diagnosis, and engineering.

In this study, we introduce the concepts of fuzzy Hopf subalgebra (FHA), fuzzy Hopf ideal (FHI) and fuzzy $H$-submodule (FHM). Some basic and essential definitions that are helpful in this paper are recalled in Section 2. The notion of fuzzy Hopf subalgebra is introduced in Section 3. In this section, we also investigate some results and examples about fuzzy Hopf subalgebra. The concept of fuzzy Hopf ideal is introduced in Section 4. In this section, we also investigate some examples and results about fuzzy Hopf ideals. The thought of fuzzy $\mathrm{H}$-submodule is offered in Section 5. In this section, we also investigate some examples and results about fuzzy $H$-submodule. Section 6 is about the advantages of our article, and Section 7 have application.

\section{Preliminaries}

In this section, we include some relevant definitions which are helpful for the reader. From now onward, except if stated otherwise, we use $H$ for Hopf algebra, $k$ for field and $Q$ for $H$-module.

Definition 2.1. [5] A HA $(H, m, u, \Delta, \varepsilon)$ is a bialgebra with antipode $S$, where $S$ is an inverse map under convolution operation.

Definition 2.2. [5] A subspace $J$ of $H$ is called Hopf subalgebra if it is subalgebra and $S(J) \subseteq J$.

Definition 2.3. [5] A $B^{c o p}$ (bialgebra having opposite comultiplication) is a co-opposite HA $H^{c o p}$ with antipode $\tilde{S}$.

Definition 2.4. [5] A subspace $I$ of $H$ is a Hopf ideal if it is a bi-ideal and $S I \subseteq I$

Definition 2.5. [5] A right $k$-space $Q$ is $H$-Hopf module of $H$ if

i. $\quad Q$ is a right $H$-module and right $H$-comodule, via $\rho: Q \rightarrow Q \otimes H$.

ii. $\rho$ is a right $H$-module map.

Definition 2.6. [13] An FS $\xi: X \rightarrow[0,1]$ is a function of a non-empty set $X$.

Definition 2.7. If $\xi$ is an FS of $X$, then the subset $\xi_{t^{\prime}}=\left\{x \in X: \xi(x) \geq t^{\prime}, t^{\prime} \in[0,1]\right\}$ is called level subsets.

Definition 2.8. [14] The intersection of two FSs $\xi$ and $\sigma$ of $X$ is also an FS of $X$ and is defined by

$$
(\xi \cap \sigma)(x)=\xi(x) \wedge \sigma(x), \text { for all } x \in X
$$

Definition 2.9. [14] The sum of two FSs $\xi$ and $\sigma$ of $X$ is defined as follows:

$$
(\xi+\sigma)(x)=\sup \{\xi(a) \wedge \sigma(b)\}, \text { for all } x \in X
$$

Definition 2.10. [20] An FS $\xi$ of $k$-vector space $V$ is called fuzzy subspace if for any $v, v^{\prime} \in V$ and $\alpha, \beta \in k$

$$
\xi\left(\alpha v+\beta v^{\prime}\right) \geq \xi(v) \wedge \xi\left(v^{\prime}\right)
$$

Definition 2.11. [22] An FS $\xi$ of a bialgebra $B$ is called fuzzy subbialgebra of $B$, if for any $b, b^{\prime} \in B$ and $\alpha, \beta \in k$, the following conditions are satisfied:

i. $\xi\left(\alpha b+\beta b^{\prime}\right) \geq \xi(b) \wedge \xi\left(b^{\prime}\right)$

ii. $\xi(b y) \geq \xi(b) \wedge \xi\left(b b^{\prime}\right)$ 
iii. $\xi(b) \leq \xi\left(b_{i 1}\right) \wedge \xi\left(b_{i 2}\right)$

Definition 2.12. [16] An FS $\xi$ of a $P$-module $Q$ is said to be a fuzzy submodule of $Q$ if for any $q, q^{\prime} \in Q$ and $p \in P$,

i. $\xi(0)=1$

ii. $\xi\left(\alpha q+\beta q^{\prime}\right) \geq \xi(q) \wedge \xi\left(q^{\prime}\right)$

iii. $\xi(p q) \geq \xi(q)$

Definition 2.13. [19] An FS $\xi$ of a $C$-comodule $Q$ and a left comodule map $\rho: Q \rightarrow C \otimes Q$ is said to be fuzzy subcomodule of $Q$, where $\rho(q)=\sum_{i=1, n} q_{i 0} \otimes q_{i 1}$ if for any $q, q^{\prime} \in Q, c \in C$, and $\alpha, \beta \in k$,

i. $\xi(0)=1$

ii. $\quad \xi\left(\alpha q+\beta q^{\prime}\right) \geq \xi(q) \wedge \xi\left(q^{\prime}\right)$

iii. $\xi(q) \leq \xi\left(q_{i 0}\right)$, for all $i$.

\section{Fuzzy Hopf Subalgebra}

In this section, the notion of FHA is proposed. Some significant results related to this concept are also discussed in it.

Definition 3.1. A FS $\xi$ of $H$ is called FHA, if for any $h, h^{\prime} \in H$ and $\alpha, \beta \in k$ it satisfies:

i. $\quad \xi\left(\alpha h+\beta h^{\prime}\right) \geq \xi(h) \wedge \xi\left(h^{\prime}\right)$

ii. $\xi\left(h h^{\prime}\right) \geq \xi(h) \wedge \xi\left(h^{\prime}\right)$

iii. $\xi(h) \leq \xi\left(h_{i 1}\right) \wedge \xi\left(h_{i 2}\right)$

iv. $\xi\left(h^{\prime}\right) \geq\left\{\begin{array}{cc}\sup \{\xi(h)\}, & \text { if } h^{\prime} \in S(H) \\ 0, & \text { if } h^{\prime} \notin S(H)\end{array}\right.$

Example 3.2. Consider the 4-dimensional $H A$

$$
H_{4}=\left\langle 1, h_{1}, h_{2}, h_{1} h_{2} \mid h_{1}{ }^{2}=1, h_{2}{ }^{2}=0, h_{2} h_{1}=-h_{1} h_{2}\right\rangle
$$

and $\xi: H_{4} \rightarrow[0,1]$ defined by

$$
\xi(a)= \begin{cases}0.4, & \text { if } a \in H_{4} \backslash\{0\} \\ 0.8, & \text { if } a=0\end{cases}
$$

Then, $\xi$ becomes an FHA of $H_{4}$.

Remark 3.3. There is no difference between FHA and fuzzy co-opposite HA because $\xi(h) \wedge \xi\left(h^{\prime}\right)=\xi\left(h^{\prime}\right) \wedge$ $\xi(h)$.

Theorem 3.4. Let $\xi$ and $\sigma$ be FHA of $H$ such that $\xi(0)=\sigma(0)$. Then, $\xi+\sigma$ is also fuzzy FHA of $H$.

PRoof. Let $h, h^{\prime} \in H$ and $\alpha, \beta \in k$. Now, we first have to show that $\xi+\sigma$ is a fuzzy subspace.

Suppose on the contrary

$$
\begin{gathered}
(\xi+\sigma)\left(\alpha h+\beta h^{\prime}\right)<(\xi+\sigma)(h) \wedge(\xi+\sigma)\left(h^{\prime}\right) \\
(\xi+\sigma)\left(\alpha h+\beta h^{\prime}\right)<(\xi+\sigma)(h)
\end{gathered}
$$

and

$$
(\xi+\sigma)\left(\alpha h+\beta h^{\prime}\right)<(\xi+\sigma)\left(h^{\prime}\right)
$$


Let $\exists t^{\prime} \in[0,1]$ such that

$$
(\xi+\sigma)\left(\alpha h+\beta h^{\prime}\right)<t^{\prime}<(\xi+\sigma)(h)
$$

and

$$
(\xi+\sigma)\left(\alpha h+\beta h^{\prime}\right)<t^{\prime}<(\xi+\sigma)\left(h^{\prime}\right)
$$

Then, $\exists h_{1}, h_{2}, h_{3}, h_{4} \in H$ with $\alpha h=h_{1}+h_{2}$

$$
\begin{gathered}
h=\left(\frac{h_{1}+h_{2}}{\alpha}\right) \text { and } \beta h^{\prime}=h_{3}+h_{4} \\
h^{\prime}=\left(\frac{h_{3}+h_{4}}{\beta}\right) \text { such that } \xi\left(\left(\frac{h_{1}+h_{2}}{\alpha}\right)\right)>t^{\prime}, \sigma\left(\left(\frac{h_{3}+h_{4}}{\beta}\right)\right)>t^{\prime}
\end{gathered}
$$

Now, we have

$$
\begin{aligned}
(\xi+\sigma)\left(\alpha h+\beta h^{\prime}\right) & =\sup _{h+h^{\prime}=m+n}\{\xi(m) \wedge \sigma(n)\} \\
& \geq \sup _{h+h^{\prime}=m+n}\left\{\xi\left(\frac{h_{1}+h_{2}}{\alpha}\right) \wedge \sigma\left(\frac{h_{3}+h_{4}}{\beta}\right)\right\} \\
& >t^{\prime}>(\xi+\sigma)\left(\alpha h+\beta h^{\prime}\right)
\end{aligned}
$$

which is contradiction. Therefore,

$$
(\xi+\sigma)\left(\alpha h+\beta h^{\prime}\right) \geq(\xi+\sigma)(h) \wedge(\xi+\sigma)\left(h^{\prime}\right)
$$

For all $h, h^{\prime} \in H$, let $h=h_{1}+h_{2}$ and $h^{\prime}=h_{3}+h_{4}$. Then, $h h^{\prime}=h_{1} h_{3}+h_{1} h_{4}+h_{2} h_{3}+h_{2} h_{4}$. Moreover,

$$
\begin{aligned}
(\xi+\sigma)\left(h h^{\prime}\right) & =\sup _{h h^{\prime}=m+n}\{\xi(m) \wedge \sigma(n)\} \\
& \geq \sin _{h h^{\prime}=h_{1} h_{3}+h_{1} h_{4}+h_{2} h_{3}+h_{2} h_{4}}\left\{\xi\left(h_{1} h_{3}+h_{1} h_{4}\right) \wedge \sigma\left(h_{2} h_{3}+h_{2} h_{4}\right)\right\} \\
& \geq \sup _{h h^{\prime}=h_{1} h_{3}+h_{1} h_{4}+h_{2} h_{3}+h_{2} h_{4}}\left\{\xi\left(h_{1} h_{3}\right) \wedge \xi\left(h_{1} h_{4}\right) \wedge \sigma\left(h_{2} h_{3}\right) \wedge \sigma\left(h_{2} h_{4}\right)\right\} \\
& \geq \sup _{h h^{\prime}=h_{1} h_{3}+h_{1} h_{4}+h_{2} h_{3}+h_{2} h_{4}}\left\{\xi\left(h_{1}\right) \wedge \xi\left(h_{3}\right) \wedge \xi\left(h_{4}\right) \wedge \sigma\left(h_{2}\right) \wedge \sigma\left(h_{3}\right) \wedge \sigma\left(h_{4}\right)\right\} \\
& \geq \sup _{h h^{\prime}=h_{1} h_{3}+h_{1} h_{4}+h_{2} h_{3}+h_{2} h_{4}}\left\{\left(\xi\left(h_{1}\right) \wedge \sigma\left(h_{2}\right)\right) \wedge\left(\xi\left(h_{3}\right) \wedge \sigma\left(h_{4}\right)\right) \wedge\left(\xi\left(h_{4}\right) \wedge \sigma\left(h_{3}\right)\right)\right\} \\
& =(\xi+\sigma)(h) \wedge(\xi+\sigma)\left(h^{\prime}\right) \wedge(\xi+\sigma)\left(h^{\prime}\right) \\
& =(\xi+\sigma)(h) \wedge(\xi+\sigma)\left(h^{\prime}\right)
\end{aligned}
$$

Furthermore, let $h=h_{1}+h_{2} \in H$. Then,

$$
\sum_{i=1, n} h_{i 1} \otimes h_{i 2}=\Delta(h)=\Delta\left(h_{1}+h_{2}\right)=\Delta\left(h_{1}\right)+\Delta\left(h_{2}\right)
$$

Here, $h_{1}=\sum_{s=1, n} h_{1(s 1)} \otimes h_{1(s 2)}$ and $h_{2}=\sum_{t=1, n} h_{2(t 1)} \otimes h_{2(t 2)}$.

Since

$$
\begin{aligned}
(\xi+\sigma)(h) & =\sup _{h=h_{1}+h_{2}}\left\{\xi\left(h_{1}\right) \wedge \sigma\left(h_{2}\right)\right\} \\
& \leq \sup _{h=h_{1}+h_{2}}\left\{\left(\xi\left(h_{1(s 1)}\right) \wedge \xi\left(h_{1(s 2)}\right)\right) \wedge\left(\sigma\left(h_{2(t 1)}\right) \wedge \sigma\left(h_{2(t 2)}\right)\right)\right\} \\
& =\sup _{h=h_{1}+h_{2}}\left\{\xi\left(h_{1(s 1)}\right) \wedge \sigma(0) \wedge \xi(0) \wedge \sigma\left(h_{2(t 1)}\right)\right\} \wedge \sup _{h=h_{1}+h_{2}}\left\{\xi\left(h_{1(s 2)}\right) \wedge \sigma(0) \wedge \xi(0) \wedge \sigma\left(h_{2(t 2)}\right)\right\}
\end{aligned}
$$




$$
\begin{aligned}
\leq & \left((\xi+\sigma)\left(h_{1(s 1)}\right) \wedge(\xi+\sigma)\left(h_{2(t 1)}\right)\right) \wedge\left((\xi+\sigma)\left(h_{1(s 2)}\right) \wedge(\xi+\sigma)\left(h_{2(t 2)}\right)\right) \\
\leq & (\xi+\sigma)\left(h_{i 1}\right) \wedge(\xi+\sigma)\left(h_{i 2}\right) \\
& (\xi+\sigma)\left(h^{\prime}\right) \geq\left\{\operatorname { s u p } _ { h \in S ^ { - 1 } ( h ^ { \prime } ) } \left\{\begin{array}{cl}
\left.\sup _{h=h_{1}+h_{2}} \xi\left(h^{1}\right) \wedge \sigma\left(h^{2}\right)\right\}, & \text { if } \mathrm{h}^{\prime} \in \mathrm{S}(\mathrm{H}) \\
0, & \text { if } h^{\prime} \notin S(H)
\end{array}\right.\right.
\end{aligned}
$$

and

$$
(\xi+\sigma)\left(h^{\prime}\right) \geq\left\{\begin{array}{cc}
\sup _{h \in S^{-1}\left(h^{\prime}\right)}(\xi+\sigma)(h), & \text { if } h^{\prime} \in S(H) \\
0, & \text { if } h^{\prime} \notin S(H)
\end{array}\right.
$$

Then $\xi+\sigma$ is FHA.

Theorem 3.5. Let $\xi$ and $\sigma$ be two FHA of $H$ then $\xi \cap \sigma$ is also FHA.

Theorem 3.6. Let $\left\{\xi_{i}: i \in N\right\}$ be any collection of an FHA of $H$ then $\cap \xi_{i}$ is also FHA.

Theorem 3.7. An FS $\xi$ of $H$ is an FHA iff the level sets $\xi_{t^{\prime}}$ are Hopf subalgebras of $H$.

Proof. Assume that $\xi$ is an FHA. Since,

$$
\begin{gathered}
\xi(h) \geq 0=t^{\prime}, \forall h \in H \\
\xi_{t^{\prime}} \neq \phi
\end{gathered}
$$

Let $h, h^{\prime} \in \xi_{t^{\prime}}, \xi(h) \geq t^{\prime}$, and $\xi\left(h^{\prime}\right) \geq t^{\prime}$. Since

$$
\xi\left(\alpha h+\beta h^{\prime}\right) \geq \xi(h) \wedge \xi\left(h^{\prime}\right) \geq t^{\prime} \wedge t^{\prime}=t^{\prime}
$$

then $\alpha h+\beta h^{\prime} \in \xi_{t^{\prime}}$. Similarly, since

$$
\xi\left(h h^{\prime}\right) \geq \xi(h) \wedge \xi\left(h^{\prime}\right) \geq t^{\prime} \wedge t^{\prime}=t^{\prime}
$$

then $h h^{\prime} \in \xi_{t^{\prime}}$.

Let $h \in H$ such that $\xi(h)=t^{\prime} \Rightarrow h \in \xi_{t^{\prime}}$ and $\Delta(h)=\sum_{i=1, n} \sum h_{i 0} \otimes h_{i 1} \Rightarrow \xi\left(h_{i 0}\right) \geq t^{\prime}$ and $\xi\left(h_{i 1}\right) \geq t^{\prime}$. Then,

$$
\xi\left(h_{i 0}\right) \wedge \xi\left(h_{i 1}\right) \geq t^{\prime} \wedge t^{\prime}=t^{\prime}=\xi(h)
$$

Let $h \in H$ such that $\xi(h)=t^{\prime} \Rightarrow h \in \xi_{t^{\prime}}$. If $h^{\prime} \in S(H)$, then,

$$
\begin{gathered}
\xi\left(h^{\prime}\right) \geq \sup _{h \in S^{-1}\left(h^{\prime}\right)} \xi(h)=t^{\prime} \\
\Rightarrow h^{\prime} \in \xi_{t^{\prime}}
\end{gathered}
$$

Hence, $\xi_{t^{\prime}}$ is Hopf subalgebra for all $t^{\prime}$.

Conversely, assume that all $\xi_{t^{\prime}}$ are Hopf subalgebras.

Let $h, h^{\prime} \in \xi_{t^{\prime}}$ and $\alpha, \beta \in k$. We may assume that

$$
\xi\left(h^{\prime}\right) \geq \xi(h)=t^{\prime}
$$

Since $h, h^{\prime} \in \xi_{t^{\prime}}$, then $\alpha h+\beta h^{\prime} \in \xi_{t^{\prime}}$. Thus,

$$
\xi\left(\alpha h+\beta h^{\prime}\right) \geq t^{\prime}=\xi(h) \wedge \xi\left(h^{\prime}\right)
$$

Since $h, h^{\prime} \in \xi_{t^{\prime}}$, then $h h^{\prime} \in \xi_{t^{\prime}}$. Thus,

$$
\xi\left(h h^{\prime}\right) \geq t^{\prime}=\xi(h) \wedge \xi\left(h^{\prime}\right)
$$


Let $h \in \xi_{t^{\prime}}$ and $\Delta(h)=\sum_{i=1, n} h_{i 0} \otimes h_{i 1}$ where $h_{i 0}, h_{i 1} \in \xi_{t^{\prime}}$. Therefore,

$$
\xi\left(h_{i 0}\right) \wedge \xi\left(h_{i 1}\right) \geq t^{\prime} \wedge t^{\prime}=t^{\prime}=\xi(h)
$$

Let $h^{\prime} \in S(H)$ and $\xi\left(h^{\prime}\right) \geq t^{\prime}$. Since $h^{\prime} \in \xi_{t^{\prime}}$ and

$$
\xi\left(h^{\prime}\right) \geq t^{\prime}=\sup _{h \in S^{-1}\left(h^{\prime}\right)} \xi(h)
$$

Then, $\xi$ is FHA.

\section{Fuzzy Hopf Ideal}

In this section, the concept of fuzzy Hopf Ideal is proposed. Some significant results of the fuzzy Hopf ideal are also studied in it.

Definition 4.1. A fuzzy subspace $\xi$ of $H$ is called fuzzy Hopf left (right) ideal if for any $h, h^{\prime} \in H$

i. $\quad \xi\left(h h^{\prime}\right) \geq \xi\left(h^{\prime}\right),\left(\xi\left(h h^{\prime}\right) \geq \xi(h)\right)$

ii. $\xi(h) \leq \xi\left(h_{i 2}\right),\left(\xi(h) \leq \xi\left(h_{i 1}\right)\right)$

iii. $\xi\left(h^{\prime}\right) \geq\left\{\begin{array}{cc}\sup _{h \in S^{-1}\left(h^{\prime}\right)}(\mathrm{h}) & \text { if } h^{\prime} \in S(H) \\ 0, & \text { if } h^{\prime} \notin S(H)\end{array}\right.$

Example 4.2. Consider $\xi: H_{4} \rightarrow[0,1]$ defined by

$$
\xi(a)=\left\{\begin{array}{lc}
0.5, & \text { if } a \in H_{4} \backslash\{0,1\} \\
0.3, & \text { if } a=1 \\
0.8, & \text { if } a=0
\end{array}\right.
$$

Then, $\xi$ becomes a fuzzy Hopf left ideal of $H_{4}$.

Remark 4.3. If $\xi$ is both right and left fuzzy Hopf ideal of $H$ then $\xi$ is called an FHI of $H$.

Theorem 4.4. Let $\xi$ and $\sigma$ be two fuzzy Hopf left (right) ideals of $H$ such that $\xi(0)=\sigma(0)$. Then, $\xi+\sigma$ is also a fuzzy Hopf left (right) ideal of $H$.

Proof. $\xi+\sigma$ is a fuzzy subspace. Let $h=h_{1}+h_{2}, h^{\prime}=h_{3}+h_{4}$. Then,

$$
h h^{\prime}=h_{1} h_{3}+h_{1} h_{4}+h_{2} h_{3}+h_{2} h_{4}
$$

Moreover,

$$
\begin{aligned}
(\xi+\sigma)\left(h h^{\prime}\right) & =\sup _{h h^{\prime}=h_{1} h_{3}+h_{1} h_{4}+h_{2} h_{3}+h_{2} h_{4}}\left\{\xi\left(h_{1} h_{3}+h_{1} h_{4}\right) \wedge \sigma\left(h_{2} h_{3}+h_{2} h_{4}\right)\right\} \\
& \geq \sup _{h h^{\prime}=h_{1} h_{3}+h_{1} h_{4}+h_{2} h_{3}+h_{2} h_{4}}\left\{\xi\left(h_{1} h_{3}\right) \wedge \xi\left(h_{1} h_{4}\right) \wedge \sigma\left(h_{2} h_{3}\right) \wedge \sigma\left(h_{2} h_{4}\right)\right\} \\
& \geq \sup _{h h^{\prime}=h_{1} h_{3}+h_{1} h_{4}+h_{2} h_{3}+h_{2} h_{4}}\left\{\xi\left(h_{3}\right) \wedge \xi\left(h_{4}\right) \wedge \sigma\left(h_{3}\right) \wedge \sigma\left(h_{4}\right)\right\} \\
& =\sup _{h h^{\prime}=h_{1} h_{3}+h_{1} h_{4}+h_{2} h_{3}+h_{2} h_{4}}\left\{\xi\left(h_{3}\right) \wedge \sigma\left(h_{4}\right) \wedge \sigma\left(h_{3}\right) \wedge \xi\left(h_{4}\right)\right\} \\
& =(\xi+\sigma)\left(h^{\prime}\right) \wedge(\xi+\sigma)\left(h^{\prime}\right) \\
& =(\xi+\sigma)\left(h^{\prime}\right)
\end{aligned}
$$

Let $h=h_{1}+h_{2} \in H$. Then,

$$
\sum_{i=1, n} h_{\mathrm{i} 1} \otimes \mathrm{h}_{\mathrm{i} 2}=\Delta(\mathrm{h})=\Delta\left(\mathrm{h}_{1}+\mathrm{h}_{2}\right)=\Delta\left(\mathrm{h}_{1}\right)+\Delta\left(\mathrm{h}_{2}\right)
$$


where, $h_{1}=\sum_{s=1, n} h_{1(s 1)} \otimes h_{1(s 2)}$ and $h_{2}=\sum_{t=1, n} h_{2(t 1)} \otimes h_{2(t 2)}$ such that

$$
\begin{aligned}
& (\xi+\sigma)(h)=\sup _{h=h_{1}+h_{2}}\left\{\xi\left(h_{1}\right) \wedge \sigma\left(h_{2}\right)\right\} \\
& \leq \sup _{h=h_{1}+h_{2}}\left\{\xi\left(h_{1(s 2)}\right) \wedge \sigma\left(h_{2(t 2)}\right)\right\} \\
& =\sup _{h=h_{1}+h_{2}}\left\{\xi\left(h_{1(s 2)}\right) \wedge \sigma(0) \wedge \xi(0) \wedge \sigma\left(h_{2(t 2)}\right)\right\} \\
& \leq(\xi+\sigma)\left(h_{1(s 2)}\right) \wedge(\xi+\sigma)\left(h_{2(t 2)}\right) \\
& \leq(\xi+\sigma)\left(h_{i 2}\right)
\end{aligned}
$$

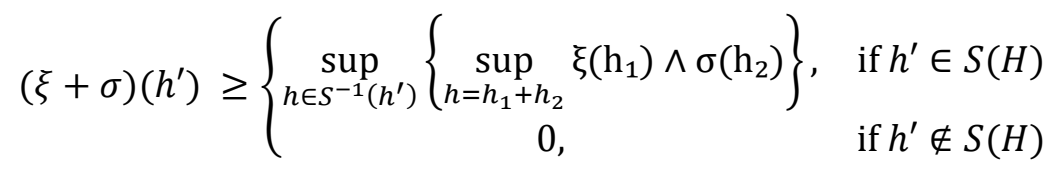

and

$$
(\xi+\sigma)\left(h^{\prime}\right) \geq\left\{\begin{array}{cc}
\sup _{h \in S^{-1}\left(h^{\prime}\right)}(\xi+\sigma)(h), & \text { if } h^{\prime} \in S(H) \\
0, & \text { if } h^{\prime} \notin S(H)
\end{array}\right.
$$

Hence, $\xi+\sigma$ is fuzzy Hopf left ideal.

Theorem 4.5. Let $\xi$ and $\sigma$ be two fuzzy Hopf left (right) ideals then $\xi \cap \sigma$ is also a fuzzy Hopf left (right) ideal.

Theorem 4.6. Let $\left\{\xi_{i}: i \in N\right\}$ be a collection of fuzzy Hopf left (right) ideals then $\cap \xi_{i}$ is also a fuzzy Hopf left (right) ideal.

\section{Fuzzy $\boldsymbol{H}$-Submodule}

In this section, the concept of fuzzy $H$-Submodule is proposed. Some significant results of fuzzy $H$-Submodule are also studied in it.

Definition 5.1. A FS $\xi$ of $Q$ is right FHM if for any $q, q^{\prime} \in Q, h \in H$ and $\alpha, \beta \in k$

i. $\xi(0)=1$

ii. $\xi\left(\alpha q+\beta q^{\prime}\right) \geq \xi(q) \wedge \xi\left(q^{\prime}\right)$

iii. $\xi(q h) \geq \xi(q)$

iv. $\xi(q) \leq \xi\left(q_{i 0}\right)$

v. $\xi\left(q^{\prime}\right) \geq\left\{\begin{array}{cc}\sup _{q \in \rho^{-1}\left(q^{\prime}\right)} \xi(\mathrm{q}), & \text { if } q^{\prime} \in \rho(Q) \\ 0, & \text { if } q^{\prime} \notin \rho(Q)\end{array}\right.$

(vi). $\xi(\rho(q) h) \geq \xi(\rho(q))$

Example 5.2. Consider $\xi: H_{4} \rightarrow[0,1]$ defined by

$$
\xi(a)=\left\{\begin{array}{cc}
0.4, & \text { if } a \in H_{4} \backslash\{0\} \\
1, & \text { if } a=0
\end{array}\right.
$$

Then, $\xi$ becomes an FHM of $H_{4}$.

Theorem 5.3. Let $\xi$ and $\sigma$ be two FHM of $Q$ such that $\xi(0)=\sigma(0)$. Then, $\xi+\sigma$ is also an FHM of $Q$. 
Proof. Let $q, q^{\prime} \in Q$ and $\alpha, \beta \in k$. Now, we have to show that $\xi+\sigma$ is a fuzzy subspace.

On the contrary, suppose that

$$
\begin{gathered}
(\xi+\sigma)\left(\alpha q+\beta q^{\prime}\right)<(\xi+\sigma)(q) \wedge(\xi+\sigma)\left(q^{\prime}\right) \\
(\xi+\sigma)\left(\alpha q+\beta q^{\prime}\right)<(\xi+\sigma)(q)
\end{gathered}
$$

and

$$
(\xi+\sigma)\left(\alpha q+\beta q^{\prime}\right)<(\xi+\sigma)\left(q^{\prime}\right)
$$

If $\exists t^{\prime} \in[0,1]$ such that

$$
(\xi+\sigma)\left(\alpha q+\beta q^{\prime}\right)<t^{\prime}<(\xi+\sigma)(q)
$$

and

$$
(\xi+\sigma)\left(\alpha q+\beta q^{\prime}\right)<t^{\prime}<(\xi+\sigma)\left(q^{\prime}\right)
$$

If $\exists q_{1}, q_{2}, q_{3}, q_{4} \in Q$ with $\alpha q=q_{1}+q_{2}$, then

$$
\begin{gathered}
q=\left(\left(q_{1}+q_{2}\right) / \alpha\right) \text { and } \beta q^{\prime}=q_{3}+q_{4} \\
q^{\prime}=\left(\frac{q_{3}+q_{4}}{\beta}\right) \text { such that } \xi\left(\left(\frac{q_{1}+q_{2}}{\alpha}\right)\right)>t^{\prime}, \sigma\left(\left(\frac{q_{3}+q_{4}}{\beta}\right)\right)>t^{\prime}
\end{gathered}
$$

Now, we have

$$
\begin{aligned}
(\xi+\sigma)\left(\alpha q+\beta q^{\prime}\right) & =\sup _{q+q^{\prime}=n+n^{\prime}}\left\{\xi(n) \wedge \sigma\left(n^{\prime}\right)\right\} \\
& \geq \sup _{q+q^{\prime}=\frac{q_{1}+q_{2}}{\alpha}+\frac{q_{3}+q_{4}}{\beta}}\left\{\xi\left(\left(\frac{q_{1}+q_{2}}{\alpha}\right)\right) \wedge \sigma\left(\left(\frac{q_{3}+q_{4}}{\beta}\right)\right)\right\} \\
& >t^{\prime}>(\xi+\sigma)\left(\alpha q+\beta q^{\prime}\right)
\end{aligned}
$$

which is a contradiction. Therefore,

$$
(\xi+\sigma)\left(\alpha q+\beta q^{\prime}\right) \geq(\xi+\sigma)(q) \wedge(\xi+\sigma)\left(q^{\prime}\right)
$$

Now, let $q \in Q$ and $h \in H$ such that

$$
\begin{aligned}
(\xi+\sigma)(q h) & =\sup _{q h=q_{1}+q_{2}}\left\{\xi\left(q_{1}\right) \wedge \sigma\left(q_{2}\right)\right\} \\
& \geq \sup _{q=\frac{q_{1}}{h}+\frac{q_{2}}{h}}\left\{\xi\left(\frac{q_{1}}{h}\right) \wedge \sigma\left(\frac{q_{2}}{h}\right)\right\} \\
& =(\xi+\sigma)(q)
\end{aligned}
$$

Let $q=q_{1}+q_{2} \in Q$. Then,

$$
\begin{aligned}
\sum_{i=1, n} q_{i 0} \otimes q_{i 1}=\rho(q) & =\rho\left(q_{1}+q_{2}\right) \\
& =\rho\left(q_{1}\right)+\rho\left(q_{2}\right) \\
& =\sum_{s=1, n} q_{1(s 0)} \otimes q_{1(s 1)}+\sum_{t=1, n} q_{2(t 0)} \otimes q_{2(t 1)}
\end{aligned}
$$

Moreover, 


$$
\begin{aligned}
(\xi+\sigma)(q) & =\sup _{q=q_{1}+q_{2}}\left\{\xi\left(q_{1}\right) \wedge \sigma\left(q_{2}\right)\right\} \\
& \leq \sup _{q=q_{1}+q_{2}}\left\{\xi\left(q_{1(s 0)}\right) \wedge \sigma\left(q_{2(t 0)}\right)\right\} \\
& =\sup _{q=q_{1}+q_{2}}\left\{\xi\left(q_{1(s 0)}\right) \wedge \sigma(0) \wedge \xi(0) \wedge \sigma\left(q_{2(t 0)}\right)\right\} \\
& =\sup _{q=q_{1}+q_{2}}\left\{\xi\left(q_{1(s 0)}\right) \wedge \sigma(0)\right\} \wedge \sup _{q=q_{1}+q_{2}} \xi(0) \wedge \sigma\left(q_{2(t 0)}\right) \\
& \leq(\xi+\sigma)\left(q_{1(s 0)}\right) \wedge(\xi+\sigma)\left(q_{2(t 0)}\right) \\
& \leq(\xi+\sigma)\left(q_{i 0}\right)
\end{aligned}
$$

and

$$
(\xi+\sigma)(0)=\sup _{0=q_{1}+q_{2}}\left\{\xi\left(q_{1}\right) \wedge \sigma\left(q_{2}\right)\right\} \geq \xi(0) \wedge \sigma(0)=1 \wedge 1=1
$$

Thus,

$$
\begin{aligned}
(\xi+\sigma)(\rho(q) h) & =\sup _{\rho(q) h=q_{1}+q_{2}}\left\{\xi\left(q_{1}\right) \wedge \sigma\left(q_{2}\right)\right\} \\
& \geq \sup _{\rho(q)=\frac{q_{1}}{h}+\frac{q_{2}}{h}}\left\{\xi\left(\frac{q_{1}}{h}\right) \wedge \sigma\left(\frac{q_{2}}{h}\right)\right\} \\
& =(\xi+\sigma)(\rho(q))
\end{aligned}
$$

Hence, $\xi+\sigma$ is FHM of $Q$.

Theorem 5.4. Let $\xi$ and $\sigma$ be two FHMs of $Q$ then $\xi \cap \sigma$ is also an FHM.

Theorem 5.5. Let $\left\{\xi_{i}: i \in N\right\}$ be a collection of FHMs of $Q$. Then $\cap \xi_{i}$ is also an FHM.

Theorem 5.6. An FS $\xi$ of $Q$ is an FHM iff the level sets $\xi_{t^{\prime}}$ are $H$-submodules of $Q$.

Proof. Assume that $\xi$ is an FHM. As $\xi(0)=1$. Therefore, $\xi_{t} \neq \varphi$. Let $q, q^{\prime} \in \xi_{t^{\prime}}$. Then,

$$
\xi(q) \geq t^{\prime} \text { and } \xi\left(q^{\prime}\right) \geq t^{\prime}
$$

Since

$$
\xi\left(\alpha q+\beta q^{\prime}\right) \geq \xi(q) \wedge \xi\left(q^{\prime}\right) \geq t^{\prime} \wedge t^{\prime}=t^{\prime}
$$

then $\alpha q+\beta q^{\prime} \in \xi_{t^{\prime}}$. Similarly, since $\xi(q h) \geq \xi(q)=t^{\prime}$ then $q h \in \xi_{t^{\prime}}$. Moreover, since $\xi\left(q_{i 0}\right) \geq \xi(q) \geq t^{\prime}$ then $x_{i 0} \in \xi_{t^{\prime}}$. Let $\rho(x) \in \xi_{t^{\prime}}$ and $h \in H$. Since

$$
\xi(\rho(q) h) \geq \xi(\rho(q)) \geq t^{\prime}
$$

then $\rho(q) h \in \xi_{t^{\prime}}$. Therefore, each $\xi_{t^{\prime}}$ is $H$-submodule of $Q$.

Conversely, assume that each $\xi_{t^{\prime}}$ is a fuzzy $H$-submodules. Let $q, q^{\prime} \in Q$ and $\alpha, \beta \in k$. We may assume that $\xi\left(q^{\prime}\right) \geq \xi(q)=t^{\prime}$. Therefore, $q, q^{\prime} \in \xi_{t^{\prime}}$ Since, $\xi_{t^{\prime}}$ is $H$-submodule of $Q$, then $\alpha q+\beta q^{\prime} \in \xi_{t^{\prime}}$. Therefore,

$$
\xi\left(\alpha q+\beta q^{\prime}\right) \geq t^{\prime}=\xi(q) \wedge \xi\left(q^{\prime}\right)
$$

Let $q \in Q$ and $h \in H$. Let $\xi(q)=t^{\prime}$. Therefore, $q \in \xi_{t^{\prime}}$ and $q h \in \xi_{t^{\prime}}$. Thus, $\xi(q h) \geq t^{\prime}=\xi(q)$.

Since $\rho(q)=\sum_{i=1, n} q_{i 0} \otimes q_{i 1}$ where $q_{i 0} \in \xi_{t^{\prime}}$, then

$$
\xi\left(q_{i 0}\right) \geq t^{\prime}=\xi(q)
$$

Moreover, 


$$
\xi(0)=\sup _{q \in Q}\{\xi(q)\}=1
$$

Let $\rho(q) \in Q$ and $h \in H$, such that $\xi(\rho(q))=t^{\prime}$. Then, $\rho(q) h \in \xi_{t^{\prime}}$

$$
\xi(\rho(q) h) \geq t^{\prime}=\xi(\rho(q))
$$

Thus, $\xi$ is an FHM.

\section{Advantages}

The importance of Hopf algebra in Quantum physics and physics cannot be rejected. Shahn Majid and other physicist and mathematician use Hopf algebra to solve many problems. By using fuzzy theory, we can solve these problems with easier. So, it is crucial to extend these concepts in uncertainty. So, in this manuscript, we proposed fuzziness on Hopf algebraic structures.

\section{Application}

Discrete gauge theory in the $2+1$ dimension arises by breaking a gauge symmetry with gauge group $G$ to discrete subgroup $H$ due to photons becoming massive w, making the guage force ultra-short ranged. Some particles are charged, and some carry flux. Charged particles carry a representation of $H$, and massive charges are ultra-short ranged. By using fuzziness, we can deal with these particles easily. Here is a simple example,

Example 7.1. Let $H=\left\{h_{1}, h_{2}, h_{3}, h_{4}\right\}$ be a Hopf algebra with antipode $S, k=\mathbb{R}, \Delta(h)=h \otimes h, \varepsilon(h)=1$,

\begin{tabular}{|c|c|c|c|c|c|c|c|c|c|c|}
\hline+ & $h_{1}$ & $h_{2}$ & $h_{3}$ & $h_{4}$ & & & $h_{1}$ & $h_{2}$ & $h_{3}$ & $h_{4}$ \\
\hline$h_{1}$ & $h_{1}$ & $h_{1}$ & $h_{1}$ & $h_{1}$ & & $h_{1}$ & $h_{1}$ & $h_{2}$ & $h_{3}$ & $h_{4}$ \\
\hline$h_{2}$ & $h_{1}$ & $h_{1}$ & $h_{1}$ & $h_{1}$ & and & $h_{2}$ & $h_{2}$ & $h_{1}$ & $h_{4}$ & $h_{3}$ \\
\hline$h_{3}$ & $h_{1}$ & $h_{1}$ & $h_{1}$ & $h_{1}$ & & $h_{3}$ & $h_{3}$ & $h_{4}$ & $h_{2}$ & $h_{1}$ \\
\hline$h_{4}$ & $h_{1}$ & $h_{1}$ & $h_{1}$ & $h_{1}$ & & $h_{4}$ & $h_{4}$ & $h_{3}$ & & $h_{2}$ \\
\hline
\end{tabular}
$S(h)=h^{-1}$,

Then, $\xi: H \rightarrow[0,1]$ defined by

$$
\xi(d)= \begin{cases}0.6, & \text { if } h=h_{1} \\ 0.4, & \text { if } h \in H /\left\{h_{1}\right\}\end{cases}
$$

is an FHA. Therefore, $h_{1}$ is the short, ranged particle.

Similarly, we can apply this uncertainty in other fields of physics, like to know the energy level of photons in non-ideal lasers etc.

\section{Conclusion}

This manuscript introduces the concept of fuzzy Hopf subalgebra, fuzzy Hopf ideal, and fuzzy H-submodule. Some properties and significant results are also studied in it. Our obtained results probably can be applied in various fields such as artificial intelligence, signal processing, multiagent systems, pattern recognition, robotics, expert systems, medical diagnosis, and engineering. Weak Hopf algebra is a Hopf algebra with a linear map that satisfy some specific conditions. In the future study, we aim to extend this concept in the field of weak Hopf algebra. 


\section{Conflict of Interest}

The authors declare no conflict of interest.

\section{References}

[1] H. Hopf, Uber die Topologie der Gruppen-Mannigfaltigkeiten und ihrer Verallgemeinerungen, Annals of Mathematics 42(1) (1941) 22-52.

[2] H. Hopf, Uber den Rang Geschlossener Liescher Gruppen, Commentarii Mathematici Helvetici 13 (1940) 119-143.

[3] E. Abe, Hopf Algebras, Cambridge University Press, 1997.

[4] M. Cohen, D. Fishman, Hopf Algebra Actions, Journal of Algebra 100 (1986) 363-379.

[5] S. Montgomery, Hopf Algebras and Their Actions on Rings, CBMS Regional Conference Series in Mathematics 82 (1993).

[6] S. Majid, Physics for Algebraists: Non-Commutative and Non-Cocomutative Hopf Algebras by a Bicrossproduct Construction, Journal of Algebra 130 (1990) 17-64.

[7] L. M. Ionescu, M. Marsalli, A Hopf Algebra Deformation Approach to Renormalization, (2003) arXiv:hep-th/0307112 2003.

[8] S. Majid, Hopf Algebra for Physics at the Plank Scale, Classical and Quantum Gravity5(12) (1988) 15871606.

[9] M. E. Sweedler, Hopf Algebras, Benjamin New-York, 1969.

[10] R. J. Blattner, M. Cohen, S. Montgomery, Cross Products and Inner Actions of Hopf Algebras, Transactions of the American Mathematical Society 298(2) (1986) 671-711.

[11] S. Montgomery, Indecomposable Coalgebras, Simple Comodules and Pointed Hopf Algebras, Proceedings of the AMS - American Mathematical Society123 (1995) 2343-2351.

[12] J. W. Milnor, J. C. Moore, On the Structures of Hopf Algebras, Annals of Mathematics 81 (1965) 211264.

[13] L. A. Zadeh, Fuzzy Sets, Information and Control 8(3) (1965) 338-353.

[14] A. Rosenfeld, Fuzzy Groups, Journal of Mathematical Analysis and Applications 35 (1971) 512-517.

[15] M. M. Zahedi, R. Ameri, Fuzzy Exact Sequence in Category of Fuzzy Modules, Journal of Fuzzy Mathematics (1994) 409-424.

[16] M. Yamin, P. K. Sharma, Group Actions on Fuzzy Modules, Applied Mathematics 7 (2016) 413-421.

[17] M. M. Zahedi, Some Results on L-fuzzy Modules, Fuzzy Sets and Systems 55 (1993) 355-361.

[18] F. Z. Pan, Fuzzy Finitely Generated Modules, Fuzzy Sets and Systems 21 (1987) 105-113.

[19] W. Chen, M. Akram, Fuzzy Subcoalgebras and Fuzzy Subcomodules, Journal of Algebra and Applied Mathematics (10) (2012) 15-32.

[20] K. S. Abdulkhalikov, M. S. Tulenbaev, U. U. Umirbaev, On Fuzzy Subalgebras, Fuzzy Sets and Systems 93 (1988) 257-262.

[21] W. Chen, Fuzzy Subcoalgebras and Duality, Bulletin of the Malaysian Mathematical Sciences Society 32(3) (2009) 283-294. 
[22] W. Chen, G. Wenxu, Fuzzy Subbialgebras and Duality, Utilitas Mathematica 87 (2012) 13-20.

[23] R. Rasuli, Norms over Fuzzy Lie Algebra, Journal of New Theory 15 (2017) 32-38.

[24] S. M. Khalil, New Category of the Fuzzy d-Algebras, Journal of Taibah University for Science 12(2) (2018) 143-149.

[25] W. Chen, Y. Guan, The Dual of Generalized Fuzzy Subspaces, Journal of Applied Mathematics Article ID 932014 (2012) 9 pages. 